\title{
1 Genetic basis of body shape variation along the benthic-pelagic axis in cichlid fishes
}

2

3 cichlid
Leah DeLorenzo ${ }^{1}$, Destiny Mathews ${ }^{1}$, A. Allyson Brandon ${ }^{1}$, Mansi Joglekar ${ }^{1}$, Aldo Carmona Baez $^{2}$, Emily C. Moore ${ }^{2,3}$, Patrick J. Ciccotto ${ }^{2,4}$, Natalie B. Roberts ${ }^{2}$, Reade B. Roberts ${ }^{2}$, and Kara E. Powder ${ }^{1 *}$

${ }^{1}$ Department of Biological Sciences, Clemson University, Clemson, SC 29634, USA.

${ }^{2}$ Department of Biological Sciences, and Genetics and Genomics Academy, North Carolina State University, Raleigh, NC 27695, USA.

${ }^{3}$ Department of Biological Sciences, University of Montana, Missoula, MT 59812, USA.

${ }^{4}$ Department of Biology, Warren Wilson College, Swannanoa, NC 28778, USA.

*Corresponding Author:

Department of Biological Sciences

Clemson University 055A Life Science Facility

190 Collings Street

Clemson, SC 29634

Tel: 864-656-3196

Email: kpowder@clemson.edu

\section{RUNNING TITLE}

Genetic basis of benthic-pelagic body shape 
ABSTRACT

31 Divergence along the benthic-pelagic axis is one of the most widespread and repeated patterns of

32 morphological variation in fishes, producing body shape diversity associated with ecology and

33 swimming mechanics. This ecological shift is also the first stage of the explosive adaptive radiation

34 of cichlid fishes in the East African Rift Lakes. We use two hybrid crosses of cichlids (Metriaclima

35 sp. $\mathrm{x}$ Aulonocara sp. and Labidochromis sp. x Labeotropheus sp., $>975$ animals total) along the

36 benthic-pelagic ecomorphological axis to determine the genetic basis of body shape

37 diversification. Using a series of both linear and geometric shape measurements, we identify 55 quantitative trait loci (QTL) that underlie various aspects of body shape variation associated with

39 benthic-pelagic divergence. These QTL are spread throughout the genome, each explain 3.0-7.2\%

40 of phenotypic variation, and are largely modular. Further, QTL are distinct both between these two

41 crosses of Lake Malawi cichlids and compared to previously identified QTL for body shape in

42 fishes such as sticklebacks. We find that body shape is controlled by many genes of small effects.

43 In all, we find that convergent benthic and pelagic body phenotypes commonly observed across

44 fish clades are most likely due to distinct genetic and molecular mechanisms.

\section{INTRODUCTION}

47 Body shape variation is common across all vertebrates and has important consequences for an 48 animal's ecology, locomotion, thermodynamics, and even speciation (Arnold, 1983, 1992; Coyne

49 \& Orr, 2004; S.T. Friedman, Price, \& Wainwright, 2021; Ruff, 1991; Schluter, 1996a, 2000;

50 Smith, Nelson-Maney, Parsons, Cooper, \& Albertson, 2015). A major axis of this morphological

51 variation is body elongation, which occurs within reptiles (Bergmann \& Irschick, 2012; Losos, 52 2009; Wiens \& Slingluff, 2001), carnivorous mammals (Law, 2019, 2021), and fishes (S. T. 
53 Friedman et al., 2020; Price et al., 2019; Ward \& Mehta, 2010). Within fishes, elongation of the

54 body is associated with a major ecomorphological divergence, the benthic-pelagic axis.

56 The benthic-pelagic axis is one of the most widespread and repeated patterns of morphological

57 variation within both marine and freshwater fishes, affecting traits such as body shape, fin position 58 and musculature, and jaw mechanics (Burns \& Sidlauskas, 2018; Cooper, Carter, Conith, Rice, \&

59 Westneat, 2017; Cooper et al., 2010; Hatfield \& Schluter, 1999; Hulsey, Roberts, Loh, Rupp, \&

60 Streelman, 2013; Kusche, Recknagel, Elmer, \& Meyer, 2014; Muschick, Indermaur, \& Salzburger,

61 2012; Price et al., 2019; Ribeiro, Davis, Rivero-Vega, Orti, \& Betancur, 2018; Robinson \& Wilson,

62 1994; Rogers \& Jamniczky, 2014; Schluter, 1996b; Walker, 1997; Willacker, Von Hippel, Wilton,

$63 \&$ Walton, 2010). This ecological and morphological divergence occurs similarly in ancient fish

64 radiations (Ribeiro et al., 2018), at the macroevolutionary level (Claverie \& Wainwright, 2014; S.

65 T. Friedman et al., 2020; Larouche et al., 2020; Price et al., 2019), and repeatedly at the

66 microevolutionary level. Benthic-pelagic body shape variation occurs during both sympatric and

67 allopatric speciation, for instance in sticklebacks (Gow, Rogers, Jackson, \& Schluter, 2008;

68 Hatfield \& Schluter, 1999; Schluter \& McPhail, 1992; Walker, 1997; Willacker et al., 2010),

69 Arctic charr (Brachmann, Parsons, Skulason, \& Ferguson, 2021), minnows (Burress, Holcomb,

70 Tan, \& Armbruster, 2017), and carp (Hollingsworth, Simons, Fordyce, \& Hulsey, 2013).

72 Benthic fishes live within rocks or shorelines and are thus in environments with variation in

73 structure and water flow. Fishes in this habitat generally have a deeper, more stout body thought

74 to be adaptive for increased maneuverability and body rotation (Webb, 1982, 1984). For instance,

75 a deeper caudal peduncle and more posterior placement of the anal and dorsal fins enable benthic 
76 fishes to have fast propulsion as they move, change direction, and adjust to varied water flow

77 patterns in their complex habitats (Koehl, 1984; Webb, 1982). This body variation can be

78 coordinated with changes in head shape, namely a shorter head region (Cooper et al., 2010). On

79 the other end of the axis, pelagic or limnetic fishes live in open waters of oceans or lakes,

80 respectively. Here, we use "pelagic" as a more generalized form of this open water habitat and

81 ecology. Pelagic fishes have repeatedly evolved a slender, more narrow fusiform body shape,

82 many times with a larger head proportion (Cooper et al., 2010; S. T. Friedman et al., 2020; Schluter

$83 \&$ McPhail, 1992). This narrower body shape is thought to enhance performance of sustained

84 swimming (“cruising”) in open water while minimizing drag (Raffini, Schneider, Franchini, Kautt,

85 \& Meyer, 2020; Webb, 1982, 1984).

87 We examine the genetic basis of this benthic-pelagic body shape variation using a textbook 88 example of adaptive radiation, cichlid fishes. Within cichlids, benthic-pelagic habitat divergence

89 is the first stage of their adaptive radiation (Kocher, 2004; Streelman \& Danley, 2003) and occurs

90 repeatedly, independently, and convergently in three expansive radiations in East African Rift

91 Lakes (Cooper et al., 2010; Hulsey, Holzman, \& Meyer, 2018; Hulsey et al., 2013; Muschick et

92 al., 2012; Ruber \& Adams, 2001) as well as smaller radiations within New World crater lakes

93 (Elmer, Kusche, Lehtonen, \& Meyer, 2010; Franchini et al., 2014; Kusche et al., 2014). Notably,

94 hybrids among difference cichlids species or even genera can be produced in the lab, enabling

95 quantitative trait loci (QTL) mapping of phenotypic variation (Powder \& Albertson, 2016).

97 We therefore capitalized on the extensive phenotypic variation and genetics of cichlids to 98 investigate the genetic basis of body shape divergence associated with the benthic-pelagic axis, a 
major axis of fish diversification. First, we determined the genetics of body shape within each cross, using species at varying points along this morphological continuum. Then, we compare if

101 the genetic intervals and mechanisms of divergence are similar between the two hybrid crosses. If

102 so, this would indicate a shared molecular control whether a fish is evolving towards a narrower

103 body shape as towards an extreme benthic species. Second, we assess the genetic architecture of

104 body shape evolution in cichlids. We ask if there are many genetic loci, each with small effects, or

105 few regions with large effects that regulate body shape. Body shape variation within stickleback

106 fishes has been shown to be due to a combination of a few QTL with large effects and many QTL

107 with small effects (Albert et al., 2008; Liu et al., 2014; Yang, Guo, Shikano, Liu, \& Merila, 2016).

108 Further, QTL for body shape and other morphological features in sticklebacks have been suggested

109 to cluster on certain chromosomes, and these "supergene" regions can influence coordinated

110 changes in phenotype (Albert et al., 2008; Liu et al., 2014; Miller et al., 2014). Finally, we ask if

111 this common and predictable morphological trajectory in fishes has a predictable genetic basis by

112 comparing QTL for body shape across benthic-pelagic divergence in multiple fish species. In other

113 words, we determine if there is parallelism in the genetics of body shape variation to accompany

114 convergent morphologies. To accomplish these goals, we utilized two hybrid crosses of Lake

115 Malawi cichlids, quantifying a suite of linear and geometric measures of body shape. We use QTL

116 mapping to identify the genetic bases of these traits, which we then compare among traits, between

117 crosses, and to studies in other non-cichlid fishes. Together, these data provide insights into the

118 genetic control of a major ecological and morphological divergence in animals. 
122 All animal care was conducted under approved IACUC protocol 14-101-O at North Carolina State

123 University. Four species of Lake Malawi cichlids were used: Aulonocara koningsi, Metriaclima

124 mbenjii, Labidochromis caeruleus, and Labeotropheus trewavasae, hereafter referred to by the

125 genus name. Though none of these species are truly pelagic, these fish serve as a phenotypic proxy

126 for various points along the primary benthic-pelagic morphological and evolutionary axis in

127 cichlid fishes (Figure 1)(Konings, 2016); it is unlikely that hybrid crosses would be viable between

128 true pelagic and benthic cichlids. Aulonocara lives within an open, sandy region, away from the

129 complex rocky habitat of the other species used here, and forages by cruising over the open sand.

130 Similarly, the Labidochromis species studied here lives in and around rocky habitats, but is non-

131 territorial and swims continuously and darts among rocks in search of invertebrate prey.

132 Metriaclima is a generalist benthic fish, and Labeotropheus represents the extreme end of benthic

133 morphology and behavior (Cooper et al., 2010; Konings, 2016). Two hybrid crosses of these

134 species were generated. The first cross came from a single Metriaclima female crossed to two

135 Aulonocara males; the inclusion of the second grandsire was inadvertent and resulted from an

136 unexpected fertilization event in these species with external fertilization. The second cross came

137 from a single Labidochromis female crossed to a single Labeotropheus male. Thus, both crosses

138 feature a fish that dominantly "cruises," a pelagic swimming tactic, versus a more benthic species.

139 For each cross, a single $\mathrm{F}_{1}$ family was generated and subsequently incrossed to produce $\mathrm{F}_{2}$ hybrid

140 mapping populations. $F_{2}$ families were raised in density-controlled aquaria with standardized

141 measured feedings until five months of age for analysis. Fish were anesthetized with buffered 100

$142 \mathrm{mg} / \mathrm{L} \mathrm{MS}-222$ for all photographs. Whole fish photographs were taken including a color standard

143 and scale bar under uniform lighting conditions in a lightbox with a mirrorless digital camera 
144 (Olympus). The sex of each animal was determined based on gonad dissection and these data were 145 omitted if there was ambiguity in gonad phenotype.

\section{Linear quantification of body shape variation}

148 We quantified various measures of body shape in 10 individuals of each parental species, 491

149 Metriaclima x Aulonocara hybrids, and 447 Labidochromis x Labeotropheus hybrids. From 150 photographs, we calculated series of linear distances (Figure 2b) using ImageJ software, including

151 standard length (snout to caudal peduncle), head length (snout to opercle), body depth (anterior

152 insertion of dorsal fin to insertion of pelvic fin), caudal peduncle width, distance between caudal

153 peduncle and anal fin insertion, width of the anal fin base, distance between anal fin and pelvic

154 fin, and pectoral fin width. ImageJ lengths in pixels were converted into centimeters using the

155 scale bar included in each picture. To remove the effects of allometry on body measures, all

156 measurements were converted into residual data by normalizing to the standard length, using a

157 dataset including both parentals and their hybrids for a single cross. Analyses including linear 158 normalization, ANOVAs, Tukey's Honest Significant Difference post-hoc tests, and correlations 159 were conducted in R.

161 Geometric quantification of body shape variation

162 Body shape variation was also quantified using geometric morphometric shape analysis.

163 Homologous anatomic landmarks were defined along the body primarily using fin insertion sites

164 as well as head landmarks such as the snout, eye, and opercle (Figure 2a). Coordinate positions of 165 all landmarks were collected from photos using the tpsDig2 software package 166 (http://www.sbmorphometrics.org/). Landmark coordinates were extracted and inputted into the R 
167 package geomorph (Adams \& Otarola-Castillo, 2013), which was used to conduct Procrustes

168 superimposition of landmarks to remove variation due to size, rotation, and position of landmarks

169 to leave variation only due to shape. The effects of allometry were removed with size correction

170 and multiple regression of shape on standard length, using a data set including both parentals and

171 their hybrids for a single cross.

172

$173 \quad$ ddRAD-sequencing

174 Genomic DNA was extracted from caudal fin tissue using DNeasy Blood and Tissue kits (Qiagen).

175 Indexed, double-digestion RADseq libraries were produced as previously described (Burford

176 Reiskind et al., 2016) and sequenced on an Illumina HiSeq with 100 bp paired end reads (North

177 Carolina State University Genomic Sciences Laboratory core facility). Raw sequencing data were

178 demultiplexed, reads were truncated to $150 \mathrm{bp}$, and low-quality reads were excluded using the

179 program process_radtags (Stacks, version 2). The demultiplexed and filtered reads were aligned

180 to the Maylandia zebra UMD2a reference genome using BWA with the mem algorithm. We used

181 the programs pstacks and cstacks (Stacks, version 1) to identify RAD markers in all the samples

182 and create a catalogue of RAD markers present in both parents of the cross. The RAD markers of

183 the progeny were subsequently matched against this catalog with the program sstacks (Stacks,

184 version 1). Genotype calls for biallelic markers with alternative alleles between the parents of the

185 cross (aa x bb markers) were generated with the program genotypes (Stacks, version 1), requiring

186 a minimum stack depth of 3 in order to export a locus in a particular individual. For the

187 Metriaclimax Aulonocara cross, markers were only used if both Aulonocara sires shared the same

188 homozygous genotype. 


\section{Linkage map}

191 The genetic map was built on the $\mathrm{R}$ statistical platform (version 4.0.3) with the package R/qtl

192 (Broman, 2009) and in-house scripts. RAD markers were sorted and binned in linkage groups

193 according to their position in the $M$. zebra UMD2a reference genome. Markers located in linkage

194 groups with more than $20 \%$ of missing data and markers located in unplaced scaffolds with more

195 than $40 \%$ of missing data were filtered out from the dataset. A chi-square test was performed with

196 the function geno.table() to detect markers with distorted segregation patterns; markers with a

197 Bonferroni-corrected p-value $<0.01$ were removed. The pairwise recombination frequencies

198 among markers were calculated, and an initial map was estimated with the functions est.rf() and

199 est.map(). Markers in linkage groups that did not show evidence of being misplaced were

200 considered to be inflating the map and removed if they increased the size of the map by at least 6

201 centiMorgans $(\mathrm{cM})$ and their flanking markers were less than $3 \mathrm{Mb}$ apart. Markers located in

202 unplaced scaffolds were integrated into a given linkage group if they had recombination frequency

203 values $<0.15$ with at least five markers from that linkage group. The remaining markers located

204 in unplaced scaffolds were removed from the map. Markers whose recombination frequency

205 profile did not match their position in the genetic map, likely due to being located in structural

206 variants or misassembled regions of the reference genome, were rearranged manually in order to

207 minimize the number of crossovers. The function calc.errorlod() was used to detect genotyping

208 errors; genotypes with a LOD score $\geq 3$ were set as missing data. The map was pruned using a

209 non-overlapping window algorithm that selected the marker in a given $2 \mathrm{cM}$ window with the least

210 amount of missing data. The final map was estimated and the maximum likelihood estimate of the

211 genotyping error rate (0.0001) was obtained with the function est.map(). The final genetic map for

212 Metriaclima x Aulonocara hybrids included 22 linkage groups, 1267 total markers, 19-127 
213 markers per linkage group, and was $1307.2 \mathrm{cM}$ in total size. The final genetic map for

214 Labidochromis x Labeotropheus hybrids included 22 linkage groups, 1180 total markers, 42-81

215 markers per linkage group, and was $1239.5 \mathrm{cM}$ in total size.

216

217 Quantitative trait loci (QTL) analysis

218 QTL mapping used multiple-QTL mapping (MQM) methods. Scripts are described in and 219 available from (Powder, 2020), follow (Jansen, 1994), and use the R/qtl package (Arends, Prins, 220 Jansen, \& Broman, 2010; Broman, Wu, Sen, \& Churchill, 2003). The process begins with a liberal 221 scan for unlinked QTL using the onescan function in R/qtl (Broman, 2009). Putative QTL with a 222 LOD approaching or above 3.0 were used to build a more rigorous statistical model. The MQM 223 method use these putative loci as cofactors during a QTL scan, verified by backward elimination.

224 The inclusion of cofactors in the final model provides more accurate detection of QTL and 225 assessment of their effects (Jansen, 1994). Statistical significance of QTL was assessed using 1000 226 permutations. For QTL peaks meeting 5\% (significance) or 10\% (suggestive) level, 95\% 227 confidence intervals were determined using Bayes analysis. Scan details such as cofactors used 228 and significance levels are reported in Table S1.

230 Markers are named based on contig and nucleotide positions in the M. zebra (zebra mbuna) 231 reference genome, M_zebra_UMD2a assembly. Names, ID numbers, and start/stop positions of 232 candidate genes within QTL intervals were extracted from the NCBI genome data viewer 233 (https://www.ncbi.nlm.nih.gov/genome/gdv) gene track for M. zebra annotation release 104. If 234 upper and lower limits of the 95\% interval were markers that mapped to unplaced scaffolds, the 235 closest marker that mapped to a placed scaffold was used instead. Gene names were compiled from 
236 the Database for Visualization and Integrated Discovery (DAVID) (Huang, Sherman, \&

237 Lempicki, 2009; Huang, Sherman, \& Lempicki, 2009) using NCBI gene ID numbers as a query.

239 Comparisons with other species

240 QTL intervals from other studies on body shape in different cichlid species (Franchini et al., 2014;

241 Fruciano et al., 2016; Navon, Olearczyk, \& Albertson, 2017), sticklebacks (Albert et al., 2008; G.

242 L. Conte et al., 2015; Liu et al., 2014; Rogers et al., 2012; Yang et al., 2016), and carp (Laghari et

243 al., 2014) were compiled, selecting for traits that were comparable with those studied here (i.e.

244 similar measures to Figure 2). Chromosomal positions were converted to comparable intervals on

245 the M. zebra (zebra mbuna) UMD2a genome assembly through a combination of manual BLAST

246 of marker sequences and based on the locations of candidate genes within intervals. Specific

247 phenotypes measured and details of conversion are detailed in Table S6.

249 RESULTS

250 Body shape variation

251 Body shape in our four parental species differs in measures such as body depth and relative head

252 proportions, which are common changes across benthic-pelagic divergence in fishes. Parental

253 species for both crosses are distinct in body shape, though not in all measures (Figures S1-S2 and

254 Table S2). For both crosses, $F_{2}$ hybrids are largely intermediate in phenotype to the two parental

255 species, though phenotypic variation in hybrids is increased and can surpass the morphological

256 range of parental species (Figures S1-S2). Thus, even for shapes with non-significant differences

257 between parental species, QTL mapping can identify genetic loci that underlie shape variation (e.g.

258 see pectoral fin width for both crosses in Figures 3-4 and Figures S1-S2). 
260 Compared to Metriaclima, Aulonocara have significantly longer head proportions and a

261 commensurate decrease in mid-body length as measured by the distance between the anal and

262 pelvic fins (Figure S1 and Table S2). These differences are also supported by geometric

263 morphometric shape analyses, which also identify coordinated changes in body shape. Together,

264 the first five principal components describe $66.1 \%$ of total shape variation (TSV) in the bodies of

265 Metriaclima sp., Aulonocara sp., and their $\mathrm{F}_{2}$ hybrids. Principal component 1 (PC1, 22.6\% TSV)

266 primarily describes variation in body depth, with positive scores associated with a deeper body

267 commonly associated with benthic fishes, such as in cichlids (Elmer et al., 2010; Hulsey et al.,

268 2018) and sticklebacks (Gow et al., 2008; Schluter \& McPhail, 1992). PC2 scores (15.5\% TSV)

269 are associated with overall proportions of head versus flank in the body. In agreement with linear

270 measurements, Aulonocara fishes are significantly associated with negative PC2 scores, which

271 characterize a body with a larger head length, a posterior shift in pectoral and pelvic fins, and

272 increased regions of the face anterior to the eye (Figure S1, Figure S3, and Table S2). Neither PC3

273 nor PC4 is significantly different between parentals. They are associated with head proportions,

274 length of the caudal region, and the position of the anal fin (PC3, 11.7\% TSV) and length of the

275 caudal region and lengths of the anal fin (PC4, 8.4\% TSV), respectively. Finally, PC5 (7.9\% TSV)

276 differs significantly between parentals, with Aulonocara fishes associated with a larger eye and

277 more posteriorly positioned dorsal fin (Figure S1, Figure S3, and Table S2).

278

279 Despite both being rock-dwelling Lake Malawi cichlids that live in sympatry (Konings, 2016),

280 Labidochromis and Labeotropheus parentals have more significant phenotypic differences than

281 the Aulonocara and Metriaclima species pair (Figure 1 and Table S2). Labeotropheus species are 
highly derived, representing an extreme benthic morphology within Lake Malawi (Cooper et al., 2010), and have significantly shorter head regions, decreased body depth, decreased caudal peduncle width, decreased anal fin width, and increased length between the caudal peduncle and the anal fin compared to Labidochromis fish (Figure S2 and Table S2). As with the other cross, these trends can be identified in the geometric morphometric shape analysis, with the first five

287 principal components explaining $64.8 \%$ of total shape variation in Labidochromis sp., parental species, nor between either parent and their $\mathrm{F}_{2}$ hybrids. However, all other PCs are

291 significantly different between Labeotropheus and Labidochromis fishes. Positive PC2 scores

292 (15.1\% TSV) are associated with Labidochromis, an increased head length, increased body depth, 293 and a longer anal fin (Figure S2, Figure S3, and Table S2). PC3 (12.9\% TSV) characterizes

294 Labidochromis fish as having a shorter distance between the caudal peduncle and anal fin, as well as a longer head region. PC4 (10.8\% TSV) primarily describes fins on the ventral side of the fish,

296 with Labidochromis and positive scores associated with a posterior shift of pectoral and pelvic fins

297 and increased length of the anal fin. Finally, PC5 (8.3\% TSV) illustrates that Labidochromis

298 species have a deeper body shape and eyes positioned more ventrally on the head (Figure S2,

299 Figure S3, and Table S2).

$301 \quad$ Sexual dimorphism in body shape

302 Differences between males and females, termed sexual dimorphism, are incredibly common across 303 species and often include differences in body size or shape (Badyaev, 2002; Fairbairn, 304 Blackenhorn, \& Szekely, 2007; Frayer \& Wolpoff, 1985; Williams \& Carroll, 2009). We therefore 
assessed differences between sexes in our two hybrid populations. Sex in Lake Malawi cichlids is genetically determined, with a diversity of sex determination loci identified (Gammerdinger \& Kocher, 2018). Within the Labidochromis x Labeotropheus cross, sex is not associated with common sex determination loci, and of the 354 animals for which sex was called, $92.9 \%$ were male. In the Metriaclima $\mathrm{x}$ Aulonocara cross, sex is solely determined by a common XY system

310 on LG7, which produces an even one-to-one sex ratio (Peterson, Cline, Moore, Roberts, \& Roberts,

311 2017; Ser, Roberts, \& Kocher, 2010). Thus, we only discuss sexual dimorphism based on 412

312 Metriaclima x Aulonocara hybrids (48.1\% male), though see Table S2 for full ANOVA analyses 313 in both crosses.

315 Overall size was the most significant body shape difference between males and females (standard

316 length, Table S2). Within the linear measures of body shape, males had significantly larger head 317 proportions, body depth, and width of the anal fin, which features egg spot pigmentation patterns 318 used during mating (Table S2). This is supported by dimorphism in geometric morphometric shape 319 analyses, with males being associated with increased body depth (positive PC1 score), a larger 320 head (negative PC2 scores), and larger anal fin (negative PC4 scores) (Figure S3, Table S2).

\section{Genetic basis of body shape variation}

323 To determine the genetic mechanisms that underlie variation in body shape, we genetically mapped 324 five PC measures of complex shape changes (Figure S3) and eight linear measures (Figure 2). We 325 did this in both the Metriaclima x Aulonocara cross and Labidochromis $\mathrm{x}$ Labeotropheus cross, 326 which represent different benthic-pelagic divergences within Lake Malawi cichlids. 
328 Thirty-four genetic intervals underlie the quantitative differences in body shape in Metriaclima $\mathrm{x}$

329 Aulonocara $\mathrm{F}_{2}$ hybrids. This includes 27 that reach 5\% statistical significance at the genome-wide

330 level and 7 that are suggestive, reaching 10\% significance levels (Figure 3, Figure S1, Figure S4,

331 Table S1). Each trait has 1-5 genetic intervals that influence phenotypic variation. QTL intervals

332 are spread throughout the genome, with 16 of 22 linkage groups each containing 1-5 loci. QTL

333 regions explain from $3.0-7.2 \%$ of the total variation in each trait (Figure S4), meaning that all body

334 shapes analyzed are controlled by many genetic loci, each with a small effect.

Despite having more phenotypically distinct parentals (Table S2), fewer QTL were identified in

338 identified, with 0-4 QTL per trait (Figure 4, Figure S2, Figure S5, Table S1). Like the other cross,

339 QTL are spread throughout the genome, with 13 of 22 linkage groups containing 1-4 QTL each.

340 Phenotypic traits are similarly complex in terms of genetics, with each QTL only accounting for

$3413.2-5.8 \%$ of shape variation (Figure S5). Even for the trait with the most variation explained (PC2

342 score in Labidochromis $\mathrm{x}$ Labeotropheus), only $21.0 \%$ of total shape is explained by the combined

343 effects of 5 QTL (Figure S5, Table S1).

345 QTL between the two crosses are largely non-overlapping both generally and for specific traits

346 (Figures 3-4, Figures S1-S5, Table S1). The linkage groups that contain "hotspots" with multiple

347 QTL in one cross are largely absent from QTL in the other cross. For example, LGs 3, 7, and 11

348 contain 4, 4, and 5 QTL respectively in the Metriaclima x Aulonocara cross; each of these LGs

349 only contain one QTL in the Labidochromis x Labeotropheus cross. Alternately, the linkage 
groups with the most QTL in the Labidochromis x Labeotropheus cross (LG4 with 3 QTL and

LG12 with 4 QTL), do not contain any QTL in the Metriaclima x Aulonocara cross.

353 This lack of common genetic signals is also the case when looking at individual traits. By and

354 large, the same trait is explained by distinct genetic intervals in each cross (Figures 3-4). In both

355 crosses, LG15 contains a QTL for anal fin width. However, the 95\% confidence intervals do not

356 overlap, residing $23 \mathrm{Mb}$ away from each other (Figures 3-4, Table $\mathrm{S} 1$ ). Thus, two distinct genetic

357 intervals control anal fin width in these crosses, but both happen to occur on the same linkage 358 group. There is also an overlap in QTL explaining PC2 scores in each cross, found on LG19. The 359 significant QTL in the Metriaclima $\mathrm{x}$ Aulonocara cross overlaps with a suggestive QTL in the

360 Labidochromis x Labeotropheus cross by 2.66 Mb (Figures 3-4, Table S1). It is notable that the

361 shapes explained by PC2 in each cross are similar, but with differences. In both cases, this variation

362 includes differences in head length. However, PC2 in the Labidochromis x Labeotropheus cross

363 also includes coordinated changes in body depth and anal fin length that do not occur in the other

364 cross (Figure S3).

366 However, there are some overlapping QTL within each cross, indicating that certain genomic 367 intervals may have pleiotropic effects on body shape (Figures 3-4, Table S1). First, three QTL 368 from the Metriaclima x Aulonocara cross overlap from 56.3-58.0 cM on LG7, contributing to 369 variation in standard length, head proportion, and PC2 shape that also describes head proportions 370 (Figure 3). Notably, all three traits are sexually dimorphic (Table S2), and the Y sex determining 371 locus is nearby at 61.4-62.9 cM (Peterson et al., 2017). Second, a QTL hotspot in the Metriaclima $372 \mathrm{x}$ Aulonocara cross occurs from 3.2-33.3 cM on LG11. Five QTL reside in this region and explain 
373 disparate variation in body shape, including head proportions (linear measure as well as PC2

374 score), the distance between the anal and pelvic fins, body depth (as PC1 score), and variation in 375 eye position and dorsal fin positioning (as PC5 score). This "hotspot" may be explained by a large,

376 previously-characterized chromosomal inversion on LG11 between Metriaclima sp. genomes and

377 Aulonocara sp. genomes (M. A. Conte et al., 2019). Finally, a QTL hotspot in the Labidochromis

378 x Labeotropheus cross occurs from 23.5-58.8 on LG12. This region underlies phenotypic variation

379 in four distinct body shapes: pectoral fin width, caudal peduncle width, body depth (as PC1 score),

380 and positioning of the pectoral and pelvic fins (as PC4 score). This region of LG12 is also notable

381 for a series of structural differences and changes in recombination rates among cichlid species (M.

382 A. Conte et al., 2019). Thus, at each interval where multiple QTL overlap, interspecific variation

383 in structural features of the genome are implicated.

385 There are no clear trends when looking at allelic effects on phenotypes in either cross (Figures S4-

386 S5, Table S1). For example, standard length in the Labidochromis x Labeotropheus cross is not

387 significantly different between species, is controlled by four QTL, and at all four of these loci the

388 derived Labeotropheus allele increases size, through a mixture of additive, overdominant, and

389 underdominant inheritance (Figure S5a). However, for PC2 shape in the same cross, which is

390 significantly higher in Labidochromis, the allele from this species only increases trait values for

391 three of the five underlying QTL (Figure S5c).

392

393 Additional work will be needed to further clarify the molecular mechanisms through which

394 phenotypic variation is generated. While some intervals have relatively few candidate genes (e.g.

395 QTL for body depth on LG14 with only 11 genes), most of our intervals contain hundreds of genes 
396 (Tables S4-S5). While it is too early to speculate on the effects of specific candidate genes in these 397 intervals, we looked for overlap between our body QTL and three genes (bmp4, lbh, and ptchl) 398 previously implicated in trophic adaptations that commonly co-occur with body shape variation in 399 cichlids (Cooper et al., 2010). Bmp4 and $l b h$ reside close together on LG19 and regulate mandible 400 length (Albertson, Streelman, Kocher, \& Yelick, 2005) and early facial patterning (Powder, 401 Cousin, McLinden, \& Albertson, 2014), respectively. These genes co-localize with a QTL for PC2 402 shape in the Metriaclima x Aulonocara cross, which largely describes changes in head length.

403 Ptchl variation produces alternate shapes in the lower jaw that represent a tradeoff between two 404 feeding mechanisms: suction feeding associated with more pelagic species and biting that is 405 common within specialized benthic species (Roberts, Hu, Albertson, \& Kocher, 2011). Ptch1 co406 localizes with the LG12 QTL hotspot in the Labidochromis $\mathrm{x}$ Labeotropheus on LG12. Though 407 this region is also associated with altered patterns of recombination, this leaves open the possibility 408 that a gene such as ptch1 may have pleiotropic effects on multiple adaptations associated with 409 benthic-pelagic divergence.

\section{Modularity of body shape variation}

412 The presence of genetic hotspots linked to multiple phenotypes suggests that a single locus may 413 have pleiotropic effects on multiple aspects of body shape variation. To directly address the 414 relationships among our measured phenotypes, we assessed correlations between all pairs of traits.

415 For both crosses, standard length was positively and strongly associated with linear measures (e.g. $416 \mathrm{r}^{2}$ values from $0.846-0.963$ in the Metriaclima $\mathrm{x}$ Aulonocara cross, Table S3). Note that

417 correlations between standard length and PC scores were conducted following a size correction.

418 After removing the effects of size, residuals for each measure were not strongly correlated with 
each other ( $\mathrm{r}^{2}$ values from -0.562 to 0.713 with a mean of 0.005 in the Metriaclima $\mathrm{x}$ Aulonocara

420 cross, and $\mathrm{r}^{2}$ values from -0.412 to 0.423 with a mean of 0.017 in the Labidochromis $\mathrm{x}$

421 Labeotropheus cross) (Table S3). This lack of correlation suggests that despite some traits having

422 overlapping genetic influences (i.e. QTL), the phenotypic patterns generated are distinct. While

423 individual linear measures did not correlate well with PC scores in the Labidochromis x

424 Labeotropheus cross, PC scores seemed to be more strongly driven by single phenotypic metrics

425 in the Metriaclima x Aulonocara cross. Specifically, PC1 score correlated with body depth (0.713),

426 PC2 score correlated with head proportions (-0.562), PC3 score correlated with the distance

427 between the caudal peduncle and anal fin (0.612), and PC4 score correlated with anal fin width (-

428 0.540) (Table S3). While these are weak correlations, this suggests that the shapes described by

429 geometric morphometrics in the Metriaclima x Aulonocara may be simpler than in the other cross,

430 and variation between these pelagic and benthic species is more modular.

\section{DISCUSSION}

\section{Evolution of an ecologically important trait}

434 We sought to understand the genetics of body shape along the benthic-pelagic axis using multiple

435 hybrid crosses of Lake Malawi cichlids and a series of both linear and geometric shape

436 measurements. We find numerous differences in body shape including body depth, head

437 proportions, caudal peduncle depth, and shifting of fin insertions that are likely to have functional

438 consequences for swimming movements. This series of changes in the body plan seen in the

439 cichlids used here mirrors variation commonly seen along the benthic-pelagic axis in fishes.

440 Further, divergence of body shape between sympatric Labidochromis and Labeotropheus

441 emphasize that while these fish encounter similar functional challenges of living in a complex 
442 benthic habitat, their distinct trophic niches are key factors driving variation in body shape.

443 Specifically, the insectivore Labidochromis has larger head proportions, increased body depth, a

444 deeper caudal peduncle, a shorter caudal region, and a longer anal fin than the algae-scraping

445 Labeotropheus (Figure S2, Table S2) (Konings, 2016). These adaptations in Labidochromis are

446 similar to fishes that use quick bursts of speed with abrupt shifts in direction (Meyers \& Belk,

447 2014; Webb, 1984), and may reflect an ability of Labidochromis to quickly maneuver and pursue

448 insect prey while Labeotropheus feed by hovering and holding steady within water flows of the

449 lake to graze on algae.

451 We then used quantitative loci mapping to identify the genetic basis of these differences in body

452 shape. We predicted that the same genetic intervals would influence body shape traits in both

453 crosses. In other words, producing body shapes associated with a benthic environment would be

454 the same regardless of relative position along the benthic-pelagic axis. However, we found that

455 QTL that control body shape variation between the open water Aulonocara and benthic

456 Metriaclima fishes are distinct from those that influence variation between a benthic generalist

457 like Labidochromis and the benthic specialist Labeotropheus (Figures 3-4, Table S1). Coupled

458 with modest effects of each QTL (3.0-7.2\% variation explained by each), this emphasizes that

459 body shape is a complex, polygenic trait and similar morphologies can be produced by multiple

460 mechanisms (a many-to-one relationship).

Distinct genetic signals regulate body shape across fishes

463 Body elongation in fishes along the benthic-pelagic axis occurs repeatedly and widely across fish

464 phylogeny, independent of time (modern versus historic) and environment (marine versus 
465 freshwater) (Burns \& Sidlauskas, 2018; S. T. Friedman et al., 2020; Gow et al., 2008; Hulsey et 466 al., 2013; Muschick et al., 2012; Ribeiro et al., 2018; Robinson \& Wilson, 1994). In "replaying 467 life's tape" (Gould, 1989), it is clear that fishes have converged on similar body shapes based on 468 ecological selection. However, it is unknown if fish species use similar genetic mechanisms to 469 achieve these predictable morphologies. Towards this goal, we compared the QTL in this study 470 with QTL identified in additional Lake Malawi species (Navon et al., 2017), crater cichlids from 471 Central America (Franchini et al., 2014), parallel radiations of sticklebacks (Albert et al., 2008 ; G.

472 L. Conte et al., 2015; Rogers et al., 2012; Yang et al., 2016), and carp (Laghari et al., 2014) (Figure

473 5, Table S6). This comes with caveats such as missing data due to unclear orthologous regions in

474 the M. zebra genome used here, the inclusion of only some fish species, and similar, yet not 475 identical measures in other studies. Despite this, our comparative approach can still identify if 476 these parallel benthic-pelagic divergences and changes in body shape are due to common genetic 477 mechanisms across multiple fish clades.

479 Overall, we find relatively minimal overlaps in QTL among our study and QTL analysis of body 480 shape in other fishes (Figures 3-5). For instance, while LG10 has been implicated in three of seven 481 previous analyses (Figure 5), neither of our crosses had a QTL on this chromosome. Likewise, 482 regions that have multiple QTL in this work (e.g. LG11 for the Metriaclima $\mathrm{x}$ Aulonocara cross 483 or LG12 for the Labidochromis x Labeotropheus cross) have not been identified in previous 484 analyses. Surprisingly, this trend even holds true for a cross within Lake Malawi (Navon et al., 485 2017), including another Labeotropheus species that is a close sister taxon to the one used here. 486 Of four QTL previously associated with body shape variation in the extreme benthic 487 Labeotropheus species, three (LG5, LG10, and LG22) show no overlap with QTL from either of 
our crosses. The fourth QTL lies on LG11, and overlaps with four QTL from the Metriaclima $\mathrm{x}$ Aulonocara cross, but not from the more similar Labidochromis x Labeotropheus cross. When some overlaps do occur, the traits do not always demonstrate a clear trend. For example, the LG19 region from 25-50cM shows an overlap of four QTL. While PC2 shape from both the Metriaclima $\mathrm{x}$ Aulonocara and Labidochromis x Labeotropheus crosses describes differences in head 493 proportions, QTL in sticklebacks includes those for unrelated traits like caudal peduncle length 494 and body depth (Figure 5, Table S6).

\section{Impacts of evolutionary history on genetic architecture and modularity}

497 Much of benthic-pelagic body shape variation in stickleback fish is caused by few genes with 498 large effects (10-20\% variation explained each) and clustered "supergene" regions that influence multiple phenotypes associated with benthic ecologies (Albert et al., 2008; Liu et al., 2014;

500 Miller et al., 2014; Yang et al., 2016). In contrast to this, we find that body shape variation in 501 Lake Malawi cichlids is due to many genes, each with a small effect (Figures S4-S5, Table S1 502 and Tables S4-5). Additionally, we find that QTL are largely spread throughout the genome 503 (Figures 3-4), and there are minimal correlations between measurements (Table S3). The largely 504 modular basis of body shape variation in cichlids has implications for evolutionary potential (i.e. 505 evolvability) and phenotypic variation (Melo, Porto, Cheverud, \& Marroig, 2016; Pigliucci \& 506 Muller, 2010; Wagner, Pavlicev, \& Cheverud, 2007). Namely, as distinct phenotypes are largely 507 controlled by independent genetic intervals in cichlids, this allows independent evolution of 508 distinct morphologies, each of which could be subject to different patterns of selection. 
510 While most of the genetic correlations identified show independent segregation and phenotypic

511 impacts, there are exceptions. One is found at the sex determination locus on LG7 in the

512 Metriaclima x Aulonocara cross, where the multiple traits mapped to this region are also

513 sexually dimorphic. Our study is unable to disentangle whether the LG7 associated trait variation

514 is sex limited (i.e. modulated by sex-specific physiology during development, where sex is

515 correlated to LG7 genotype), or results from allelic variation that is in linkage with the sex

516 determination locus. If future studies indicate the latter, this would support the hypothesis that

517 sexual dimorphism in body morphology evolves from accumulation of sexually antagonistic

518 alleles at sex determination loci, as has been suggested for sexual dimorphism in pigmentation

519 (Albertson et al., 2014; Roberts, Ser, \& Kocher, 2009). Two other hotspots containing multiple

520 trait QTL were found, one on LG11 in the Metriaclima x Aulonocara cross, and the other on

521 LG12 in the Labidochromis $x$ Labeotropheus cross. There is clear evidence for an interspecific

522 inversion at LG11 between Metriaclima and Aulonocara, and recombination patterns among

523 different hybrid crosses suggest that LG12 has significant variation in structure among Lake

524 Malawi cichlids (M. A. Conte et al., 2019). Inversions and other structural variants can strongly

525 suppress recombination, and this could support the evolutionary accumulation of complimentary

526 adaptive alleles at multiple loci within broad haplotypes. Similar roles for inversions have been

527 suggested in the parallel adaptation of sticklebacks (Jones et al., 2012), including the predictable

528 fixation of certain inversion haplotypes within freshwater populations (Roesti, Kueng, Moser, \&

529 Berner, 2015). Within the Lake Malawi radiation, where occasional interspecific hybridization

530 occurs and likely supports evolution, inversions may preserve combinations of alleles that drive

531 multiple, distinct traits in the same direction along the benthic-pelagic axis, while avoiding

532 discordant phenotypes. Our observations indicate a need for additional work dissecting genetic 
533 variation at structural variants. This could determine whether multiple genes are involved, or

534 whether the hotspots represent the pleiotropic effects of a single gene that happens to lie within a

535 structural variant.

537 This different genetic architecture and pattern of modularity within stickleback and cichlid fishes

538 is likely due to their different evolutionary histories and ancestral states. Specifically, ancestral

539 sticklebacks are marine, pelagic morphs that have a strong selective pressure towards a freshwater,

540 benthic form when migrating into small lakes created by glacial retreat (Schluter \& McPhail, 1992;

541 Walker, 1997). On the other hand, cichlids evolved from a small group of generalist, riverine

542 species (Malinsky et al., 2018) in sympatry towards multiple adaptive peaks. While benthic-

543 pelagic habitat divergence is the first stage of the cichlid radiation and influences patterns of

544 speciation, this is only one of many selective pressures (Kocher, 2004; Streelman \& Danley, 2003).

545 Further, the cichlid "hybrid swarm" has extensive shared genetic variation and ongoing

546 hybridization among fish at varying points along the benthic-pelagic axis, which further influences

547 the genetic architecture, modularity, and evolutionary potential of morphological variation in

548 cichlids (Brawand et al., 2014; Malinsky et al., 2018).

\section{CONCLUSIONS}

551 Body elongation is common across animals. In fishes, the benthic-pelagic ecomorphological axis 552 is a major source of phenotypic variation, encompassing a suite of body shape phenotypes. We

553 show here that this convergent phenotype is most likely due to distinct molecular signals in

554 different fish clades or even at different points along a morphological continuum in a single

555 radiation. Through comparison of genetic mapping results in two hybrid crosses, we show here 
that even the closely related cichlid species examined have distinct genetic architectures for this

convergent trait. The genetic loci we identify here additionally serve as candidates to understand

the molecular origins of an ecologically relevant trait, body shape variation.

\section{ACKNOWLEDGEMENTS}

561 We thank Dr. Samantha Price for useful discussion and Dr. Daniela Almeida for useful comments

on initial manuscripts. This work was supported by NSF CAREER \#1942178 (KEP), NIH

P20GM121342 (KEP), and NIH R15DE029945 (KEP), NSF IOS-1456765 (RBR), and an Arnold

and Mabel Beckman Institute Young Investigator Award (RBR).

\section{REFERENCES}

Adams, D., \& Otarola-Castillo, E. (2013). geomorph: an r package for the collection and analysis of geometric morphometric shape data. Methods Ecol Evol, 4(4), 393-399.

Albert, A. Y., Sawaya, S., Vines, T. H., Knecht, A. K., Miller, C. T., Summers, B. R., ... Schluter, D. (2008). The genetics of adaptive shape shift in stickleback: pleiotropy and effect size. Evolution, 62(1), 76-85. doi:10.1111/j.1558-5646.2007.00259.x

Albertson, R. C., Powder, K. E., Hu, Y., Coyle, K. P., Roberts, R. B., \& Parsons, K. J. (2014). Genetic basis of continuous variation in the levels and modular inheritance of pigmentation in cichlid fishes. Mol Ecol, 23(21), 5135-5150. doi:10.1111/mec.12900

Albertson, R. C., Streelman, J. T., Kocher, T. D., \& Yelick, P. C. (2005). Integration and evolution of the cichlid mandible: the molecular basis of alternate feeding strategies. Proc Natl Acad Sci U S A, 102(45), 16287-16292. doi:10.1073/pnas.0506649102

Arends, D., Prins, P., Jansen, R. C., \& Broman, K. W. (2010). R/qtl: high-throughput multiple QTL mapping. Bioinformatics, 26(23), 2990-2992. doi:10.1093/bioinformatics/btq565

Arnold, S. (1983). Morphology, Performance and Fitness. Amer Zool, 23, 347-361.

Arnold, S. (1992). Constraints on phenotypic evolution. Am Nat, 140, S85-107.

Badyaev, A. V. (2002). Growing apart: an ontogenetic perspective on the evolution of sexual size dimorphism. Trends in Ecology \& Evolution, 17(8), 369-378.

Bergmann, P. J., \& Irschick, D. J. (2012). Vertebral evolution and the diversification of squamate reptiles. Evolution, 66(4), 1044-1058. doi:10.1111/j.1558-5646.2011.01491.x

Brachmann, M. K., Parsons, K., Skulason, S., \& Ferguson, M. M. (2021). The interaction of resource use and gene flow on the

phenotypic divergence of benthic and pelagic morphs of 
Icelandic Arctic charr (Salvelinus alpinus). Ecol Evol, 11, 7315-7334.

Brawand, D., Wagner, C. E., Li, Y. I., Malinsky, M., Keller, I., Fan, S., . . Di Palma, F. (2014). The genomic substrate for adaptive radiation in African cichlid fish. Nature, 513(7518), 375-381. doi:10.1038/nature13726

Broman, K. W. (2009). A guide to QTL mapping with R/qtl. New York, NY: Springer.

Broman, K. W., Wu, H., Sen, S., \& Churchill, G. A. (2003). R/qtl: QTL mapping in experimental crosses. Bioinformatics, 19(7), 889-890. doi:10.1093/bioinformatics/btg112

Burford Reiskind, M. O., Coyle, K., Daniels, H. V., Labadie, P., Reiskind, M. H., Roberts, N. B., ... Schaff, J. (2016). Development of a universal double-digest RAD sequencing approach for a group of non-model, ecologically and economically important insect and fish taxa. Molecular Ecology Resources. doi:doi: 10.1111/1755-0998.12527

Burns, M. D., \& Sidlauskas, B. L. (2018). Ancient and contingent body shape

diversification in a hyperdiverse continental

fish radiation. Evolution, 73(3), 569-587.

Burress, E. D., Holcomb, J. M., Tan, M., \& Armbruster, J. W. (2017). Ecological diversification associated with the benthic-to-pelagic transition by North American minnows. J Evol Biol, 30(3), 549-560. doi:10.1111/jeb.13024

Claverie, T., \& Wainwright, P. C. (2014). A morphospace for reef fishes: elongation is the dominant axis of body shape evolution. PLoS One, 9(11), e112732. doi:10.1371/journal.pone.0112732

Conte, G. L., Arnegard, M. E., Best, J., Chan, Y. F., Jones, F. C., Kingsley, D. M., . . Peichel, C. L. (2015). Extent of QTL Reuse During Repeated Phenotypic Divergence of Sympatric Threespine Stickleback. Genetics, 201(3), 1189-1200. doi:10.1534/genetics.115.182550

Conte, M. A., Joshi, R., Moore, E. C., Nandamuri, S. P., Gammerdinger, W. J., Roberts, R. B., . . . Kocher, T. D. (2019). Chromosome-scale assemblies reveal the structural evolution of African cichlid genomes. Gigascience, 8(4). doi:10.1093/gigascience/giz030

Cooper, W. J., Carter, C. B., Conith, A. J., Rice, A. N., \& Westneat, M. W. (2017). The evolution of jaw protrusion mechanics is tightly coupled to bentho-pelagic divergence in damselfishes (Pomacentridae). J Exp Biol, 220(Pt 4), 652-666. doi:10.1242/jeb.143115

Cooper, W. J., Parsons, K., McIntyre, A., Kern, B., McGee-Moore, A., \& Albertson, R. C. (2010). Bentho-pelagic divergence of cichlid feeding architecture was prodigious and consistent during multiple adaptive radiations within African rift-lakes. PLoS One, 5(3), e9551. doi:10.1371/journal.pone.0009551

Coyne, J., \& Orr, H. (2004). Speciation. Sunderland, MA: Sinauer Associates.

Elmer, K. R., Kusche, H., Lehtonen, T. K., \& Meyer, A. (2010). Local variation and parallel evolution: morphological and genetic diversity across a species complex of neotropical crater lake cichlid fishes. Philos Trans R Soc Lond B Biol Sci, 365(1547), 1763-1782. doi:10.1098/rstb.2009.0271

Fairbairn, D., Blackenhorn, W., \& Szekely, T. (2007). Sex, size and gender roles. Evolutionary Studies of Sexual Size Dimorphism. New York: Oxford University Press.

Franchini, P., Fruciano, C., Spreitzer, M. L., Jones, J. C., Elmer, K. R., Henning, F., \& Meyer, A. (2014). Genomic architecture of ecologically divergent body shape in a pair of sympatric crater lake cichlid fishes. Mol Ecol, 23(7), 1828-1845. doi:10.1111/mec.12590

Frayer, D. W., \& Wolpoff, M. H. (1985). Sexual Dimorphism. Annual Reviews Anthropology, 14, 429-473. 
Friedman, S. T., Price, S. A., Corn, K. A., Larouche, O., Martinez, C. M., \& Wainwright, P. C. (2020). Body shape diversification along the benthic-pelagic axis in marine fishes. Proc Biol Sci, 287(1931), 20201053. doi:10.1098/rspb.2020.1053

Friedman, S. T., Price, S. A., \& Wainwright, P. C. (2021). The Effect of Locomotion Mode on Body Shape Evolution in Teleost Fishes. Integrative Organismal Biology, 3(1), obab016.

Fruciano, C., Franchini, P., Kovacova, V., Elmer, K. R., Henning, F., \& Meyer, A. (2016). Genetic linkage of distinct adaptive traits in sympatrically speciating crater lake cichlid fish. Nat Commun, 7, 12736. doi:10.1038/ncomms 12736

Gammerdinger, W. J., \& Kocher, T. D. (2018). Unusual Diversity of Sex Chromosomes in African Cichlid Fishes. Genes (Basel), 9(10). doi:10.3390/genes9100480

Gould, S. J. (1989). Wonderful Life: The Burgess Shale and the Nature of History. New York, New York: W. W. Norton \& Company.

Gow, J., Rogers, S., Jackson, M., \& Schluter, D. (2008). Ecological predictions lead to the discovery of a benthic-limnetic sympatric species pair of

threespine stickleback in Little Quarry Lake, British Columbia. Can J Zool, 86, 564-571. doi:doi:10.1139/Z08-032

Hatfield, T., \& Schluter, D. (1999). Ecological Speciation in Sticklebacks: EnvironmentDependent Hybrid Fitness. Evolution, 53(3), 866-873. doi:10.1111/j.15585646.1999.tb05380.x

Hollingsworth, P. R., Jr., Simons, A. M., Fordyce, J. A., \& Hulsey, C. D. (2013). Explosive diversification following a benthic to pelagic shift in freshwater fishes. BMC Evol Biol, 13, 272. doi:10.1186/1471-2148-13-272

Huang, D. W., Sherman, B. T., \& Lempicki, R. A. (2009). Bioinformatics enrichment tools: paths toward the comprehensive functional analysis of large gene lists. Nucleic Acids Res, 37(1), 1-13. doi:10.1093/nar/gkn923

Huang, D. W., Sherman, B. T., \& Lempicki, R. A. (2009). Systematic and integrative analysis of large gene lists using DAVID bioinformatics resources. Nat Protoc, 4(1), 44-57. doi:10.1038/nprot.2008.211

Hulsey, C. D., Holzman, R., \& Meyer, A. (2018). Dissecting a potential spandrel of adaptive radiation: Body depth and pectoral fin ecomorphology coevolve in Lake Malawi cichlid fishes. Ecol Evol, 8(23), 11945-11953. doi:10.1002/ece3.4651

Hulsey, C. D., Roberts, R. J., Loh, Y. H., Rupp, M. F., \& Streelman, J. T. (2013). Lake Malawi cichlid evolution along a benthic/limnetic axis. Ecol Evol, 3(7), 2262-2272. doi:10.1002/ece3.633

Jansen, R. C. (1994). Controlling the type I and type II errors in mapping quantitative trait loci. Genetics, 138(3), 871-881.

Jones, F. C., Grabherr, M. G., Chan, Y. F., Russell, P., Mauceli, E., Johnson, J., . . Kingsley, D. M. (2012). The genomic basis of adaptive evolution in threespine sticklebacks. Nature, 484(7392), 55-61. doi:10.1038/nature10944

Kocher, T. D. (2004). Adaptive evolution and explosive speciation: the cichlid fish model. Nat Rev Genet, 5(4), 288-298. doi:10.1038/nrg1316

Koehl, M. (1984). How do benthic organisms withstand moving water? Amer Zool, 24, 57-70.

Konings, A. (2016). Malawi cichlids in their natural habitat (5th ed.): Cichlid Press.

Kusche, H., Recknagel, H., Elmer, K. R., \& Meyer, A. (2014). Crater lake cichlids individually specialize along the benthic-limnetic axis. Ecol Evol, 4(7), 1127-1139. doi:10.1002/ece3.1015 
Laghari, M. Y., Lashari, P., Zhang, X., Xu, P., Narejo, N. T., Liu, Y., . . Sun, X. (2014). Mapping QTLs for swimming ability related traits in Cyprinus carpio L. Mar Biotechnol (NY), 16(6), 629-637. doi:10.1007/s10126-014-9578-8

Larouche, O., Benton, B., Corn, K. A., Friedman, S. T., Gross, D., Iwan, M., . . Price, S. A. (2020). Reef-associated fishes have more maneuverable body shapes at a macroevolutionary scale. Coral Reefs, 39, 1427-1439.

Law, C. J. (2019). Evolutionary shifts in extant mustelid (Mustelidae: Carnivora) cranial shape, body size and body shape coincide with the Mid-Miocene Climate Transition. Biol Lett, 15(5), 20190155. doi:10.1098/rsbl.2019.0155

Law, C. J. (2021). Evolutionary and morphological patterns underlying carnivoran body shape diversity. Evolution, 75(2), 365-375. doi:10.1111/evo.14143

Liu, J., Shikano, T., Leinonen, T., Cano, J. M., Li, M. H., \& Merila, J. (2014). Identification of major and minor QTL for ecologically important morphological traits in three-spined sticklebacks (Gasterosteus aculeatus). G3 (Bethesda), 4(4), 595-604. doi:10.1534/g3.114.010389

Losos, J. (2009). Lizards in an Evolutionary Tree: Ecology and Adaptive Radiation of Anoles. Berkeley, CA: University of California Press.

Malinsky, M., Svardal, H., Tyers, A. M., Miska, E. A., Genner, M. J., Turner, G. F., \& Durbin, R. (2018). Whole-genome sequences of Malawi cichlids reveal multiple radiations interconnected by gene flow. Nat Ecol Evol, 2(12), 1940-1955. doi:10.1038/s41559-0180717-x

Melo, D., Porto, A., Cheverud, J. M., \& Marroig, G. (2016). Modularity: genes, development and evolution. Annu Rev Ecol Evol Syst, 47, 463-486. doi:10.1146/annurev-ecolsys-121415032409

Meyers, P. J., \& Belk, M. C. (2014). Shape variation in a benthic stream fish across flow regimes. Hydrobiologia, 738, 147-154.

Miller, C. T., Glazer, A. M., Summers, B. R., Blackman, B. K., Norman, A. R., Shapiro, M. D., . . . Kingsley, D. M. (2014). Modular skeletal evolution in sticklebacks is controlled by additive and clustered quantitative trait Loci. Genetics, 197(1), 405-420. doi:10.1534/genetics.114.162420

Muschick, M., Indermaur, A., \& Salzburger, W. (2012). Convergent evolution within an adaptive radiation of cichlid fishes. Curr Biol, 22(24), 2362-2368. doi:10.1016/j.cub.2012.10.048

Navon, D., Olearczyk, N., \& Albertson, R. C. (2017). Genetic and developmental basis for fin shape variation in African cichlid fishes. Mol Ecol, 26(1), 291-303. doi: $10.1111 / \mathrm{mec} .13905$

Peterson, E. N., Cline, M. E., Moore, E. C., Roberts, N. B., \& Roberts, R. B. (2017). Genetic sex determination in Astatotilapia calliptera, a prototype species for the Lake Malawi cichlid radiation. Naturwissenschaften, 104(5-6), 41. doi:10.1007/s00114-017-1462-8

Pigliucci, M., \& Muller, G. B. (2010). Evolution, the Extended Synthesis. Cambridge, MA: MIT Press.

Powder, K. E. (2020). Quantitative Trait Loci (QTL) Mapping. Methods Mol Biol, 2082, 211-229. doi:10.1007/978-1-0716-0026-9_15

Powder, K. E., \& Albertson, R. C. (2016). Cichlid fishes as a model to understand normal and clinical craniofacial variation. Dev Biol, 415(2), 338-346. doi:10.1016/j.ydbio.2015.12.018

Powder, K. E., Cousin, H., McLinden, G. P., \& Albertson, R. C. (2014). A nonsynonymous mutation in the transcriptional regulator $\mathrm{lbh}$ is associated with cichlid craniofacial 
adaptation and neural crest cell development. Mol Biol Evol, 31(12), 3113-3124. doi:10.1093/molbev/msu267

Price, S. A., Friedman, S. T., Corn, K. A., Martinez, C. M., Larouche, O., \& Wainwright, P. C. (2019). Building a Body Shape Morphospace of Teleostean Fishes. Integr Comp Biol, 59(3), 716-730. doi:10.1093/icb/icz115

Raffini, F., Schneider, R. F., Franchini, P., Kautt, A. F., \& Meyer, A. (2020). Diving into divergence: Differentiation in swimming performances, physiology and gene expression between locally-adapted sympatric cichlid fishes. Mol Ecol, 29(7), 1219-1234. doi:10.1111/mec.15304

Ribeiro, E., Davis, A. M., Rivero-Vega, R. A., Orti, G., \& Betancur, R. R. (2018). Post-Cretaceous bursts of evolution along the benthic-pelagic axis in marine fishes. Proc Biol Sci, 285(1893), 20182010. doi:10.1098/rspb.2018.2010

Roberts, R. B., Hu, Y., Albertson, R. C., \& Kocher, T. D. (2011). Craniofacial divergence and ongoing adaptation via the hedgehog pathway. Proc Natl Acad Sci US A, 108(32), 1319413199. doi:10.1073/pnas.1018456108

Roberts, R. B., Ser, J. R., \& Kocher, T. D. (2009). Sexual conflict resolved by invasion of a novel sex determiner in Lake Malawi cichlid fishes. Science, 326(5955), 998-1001. doi:10.1126/science.1174705

Robinson, B., \& Wilson, D. (1994). Character Release and Displacement in Fishes: A Neglected Literature. Am Nat, 144(4), 596-627.

Roesti, M., Kueng, B., Moser, D., \& Berner, D. (2015). The genomics of ecological vicariance in threespine stickleback fish. Nat Commun, 6, 8767. doi:10.1038/ncomms9767

Rogers, S. M., \& Jamniczky, H. A. (2014). The shape of things to come in the study of the origin of species? Mol Ecol, 23, 1650-1652.

Rogers, S. M., Tamkee, P., Summers, B., Balabahadra, S., Marks, M., Kingsley, D. M., \& Schluter, D. (2012). Genetic signature of adaptive peak shift in threespine stickleback. Evolution, 66(8), 2439-2450. doi:10.1111/j.1558-5646.2012.01622.x

Ruber, L., \& Adams, D. (2001). Evolutionary convergence of body shape and trophic morphology in cichlids from Lake Tanganyika. J Evol Biol, 14(2), 325-332.

Ruff, C. (1991). Climate and body shape in hominid evolution. J Human Evol, 21(2), 81-105.

Schluter, D. (1996a). Ecological causes of adaptive radiation. Am Nat, 148, S40-S64.

Schluter, D. (1996b). Ecological speciation in postglacial fishes. Philos Trans $R$ Soc Lond B Biol Sci, 341, 807-814.

Schluter, D. (2000). The Ecology of Adaptive Radiation: Oxford University Press.

Schluter, D., \& McPhail, J. (1992). Ecological character displacement and speciation in sticklebacks. Am Nat, 140, 85-108. doi:doi:10.1086/285404

Ser, J. R., Roberts, R. B., \& Kocher, T. D. (2010). Multiple interacting loci control sex determination in lake Malawi cichlid fish. Evolution, 64(2), 486-501. doi:10.1111/j.15585646.2009.00871.x

Smith, A., Nelson-Maney, N., Parsons, K. J., Cooper, W., \& Albertson, R. C. (2015). Body Shape Evolution in Sunfishes: Divergent Paths to Accelerated Rates of Speciation in the Centrarchidae. Evol Biol, 42, 283-295.

Streelman, J. T., \& Danley, P. (2003). The stages of vertebrate evolutionary radiation. Trends in Ecology \& Evolution, 18(3), 126-131.

Wagner, G. P., Pavlicev, M., \& Cheverud, J. M. (2007). The road to modularity. Nat Rev Genet, 8(12), 921-931. doi:10.1038/nrg2267 
Walker, J. A. (1997). Ecological morphology of lacustrine threespine stickleback Gasterosteus aculeatus

(Gasterosteidae) body shape. Biol J Linnean Soc, 61, 3-59.

Ward, A. B., \& Mehta, R. S. (2010). Axial elongation in fishes: using morphological approaches to elucidate developmental mechanisms in studying body shape. Integr Comp Biol, 50(6), 1106-1119. doi:10.1093/icb/icq029

Webb, P. (1982). Locomotor patterns in the evolution of actinopterygian fishes. Amer Zool, 22, 329-342.

Webb, P. (1984). Body form, locomotion and foraging in aquatic vertebrates. Amer Zool, 24, 107120.

Wiens, J., \& Slingluff, J. (2001). How lizards turn into snakes: a phylogenetic analysis of bodyform evolution in anguid lizards. Evolution, 55(11), 2303-2318.

Willacker, J. J., Von Hippel, F. A., Wilton, P. R., \& Walton, K. M. (2010). Classification of threespine stickleback along the benthic-limnetic axis. Biol J Linnean Soc, 101, 595-608.

Williams, T. M., \& Carroll, S. B. (2009). Genetic and molecular insights into the development and evolution of sexual dimorphism. Nat Rev Genet, 10(11), 797-804. doi:10.1038/nrg2687

Yang, J., Guo, B., Shikano, T., Liu, X., \& Merila, J. (2016). Quantitative trait locus analysis of body shape divergence in nine-spined sticklebacks based on high-density SNP-panel. Sci Rep, 6, 26632. doi:10.1038/srep26632

DATA ACCESSIBILITY: Raw data files are available at Dryad [link to be provided prior to publication]. These files include phenotypic measures, TPS files for geometric morphometric analysis, and genotypes used for quantitative trait loci mapping.

\section{AUTHOR CONTRIBUTIONS}

KEP and RBR designed the research. ACB, ECM, PJC, and NBR performed animal husbandry, photography, and collections. NBR prepared sequencing libraries. KEP, DM, and MJ performed phenotypic measurements. KEP, LD, ECM, ACB, and RBR analyzed data. KEP, LD, and AAB wrote the paper with edits from all authors.

\section{FIGURES WITH LEGENDS}




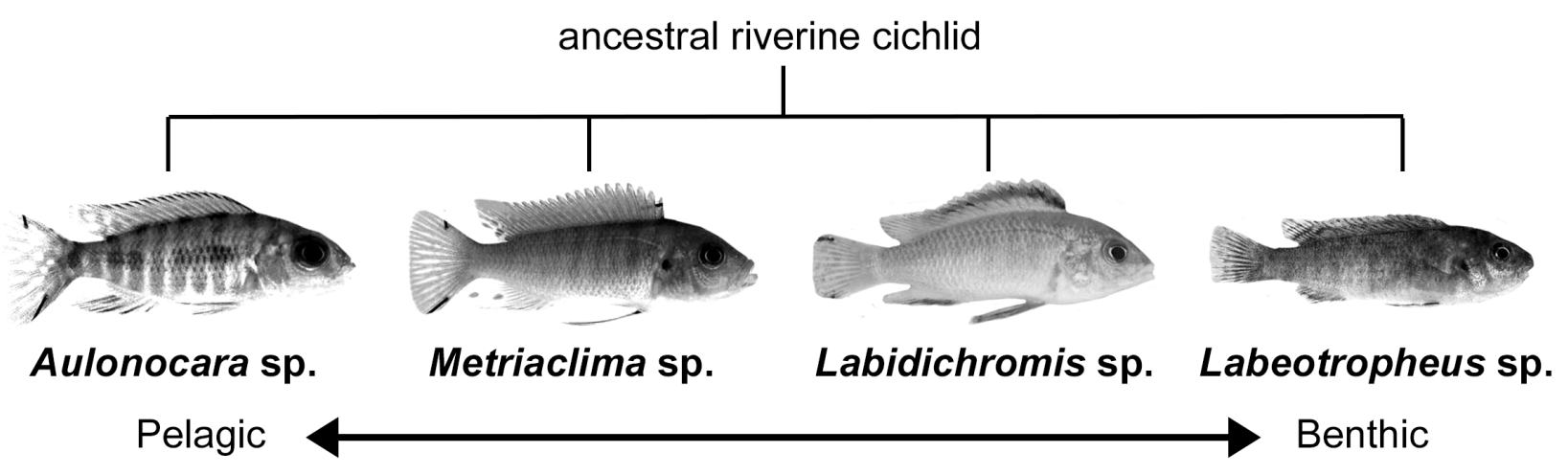

805 Figure 1. Parental species represent various points along the benthic-pelagic

806 ecomorphological axis of Lake Malawi cichlids. Two $\mathrm{F}_{2}$ hybrid populations were generated. The

807 benthic Metriaclima was crossed to Aulonocara, which lives in open sandy areas. Another cross

808 was within benthic species, with Labidochromis crossed to the extreme benthic specialist

809 Labeotropheus. 


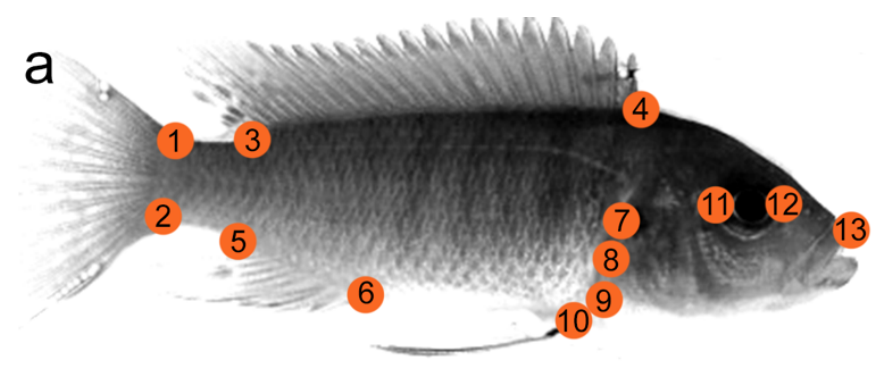

1-2: Dorsal and ventral edges of caudal peduncle 3-4: Anterior and posterior insertions of the dorsal fin 5-6: Anterior and posterior insertions of the anal fin 7: Posterior point of the opercle bone 8-9: Dorsal and ventral insertions of the pectoral fin 10: Insertion of the pelvic fin

11-12: Anterior and posterior edges of the eye 13: Tip of the snout
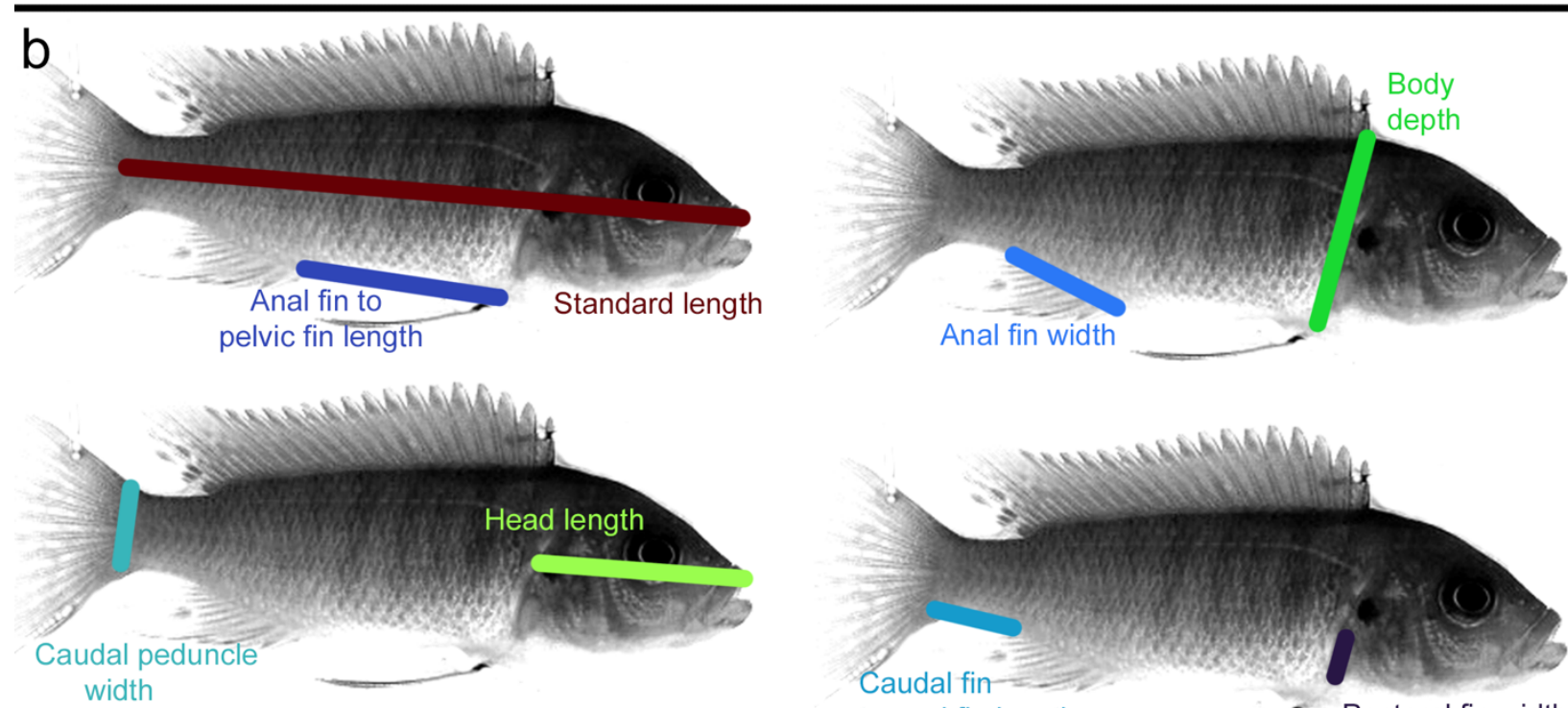

812 Figure 2. Measures used to assess body shape. (a) Geometric and (b) linear measures were used

813 to assess body shape changes commonly seen along benthic-pelagic morphological divergence in

814 fishes. Phenotypes are color-coded the same throughout figures. 


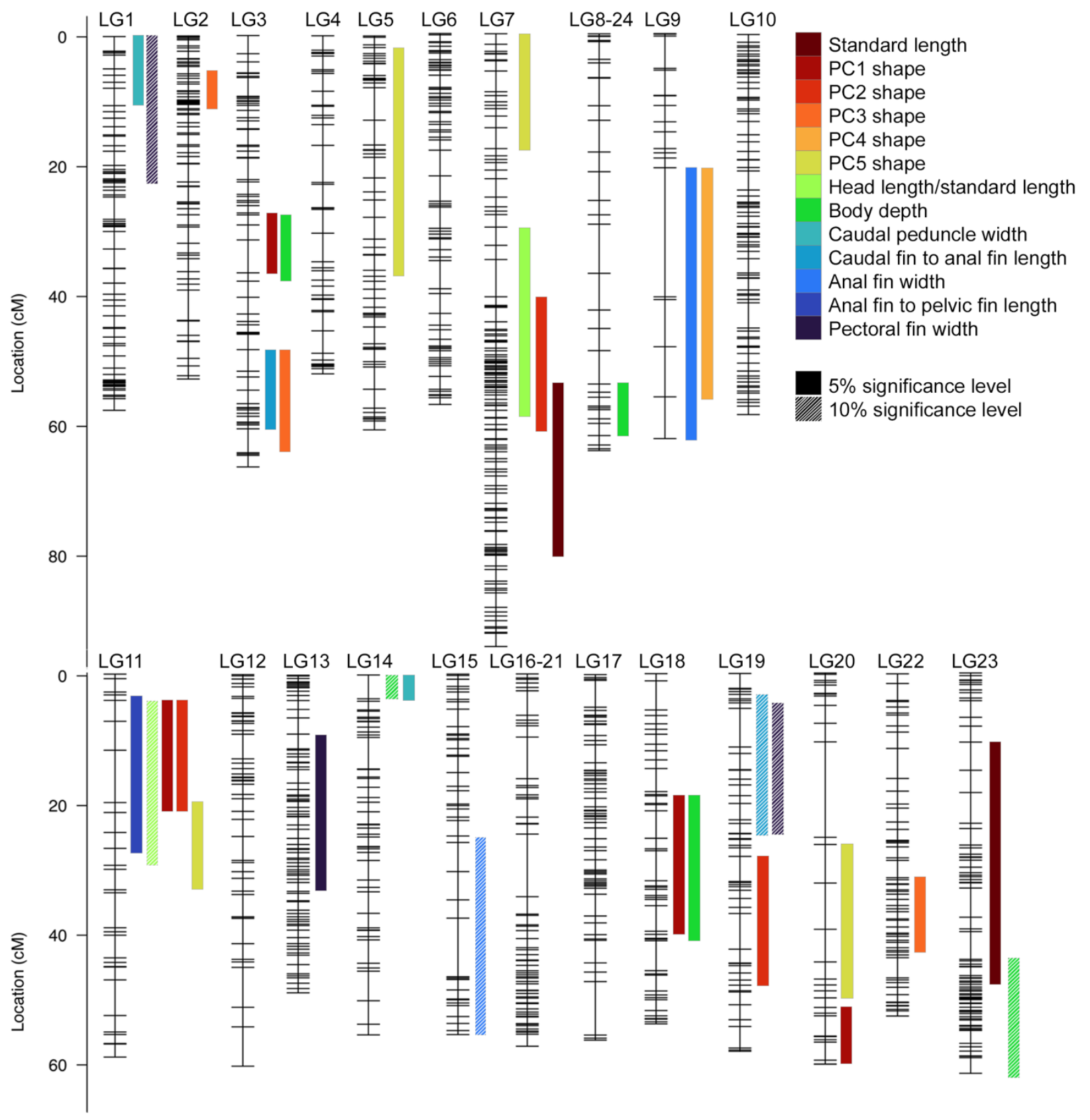

818 Figure 3. Quantitative trait loci (QTL) mapping identifies 34 intervals associated with

819 changes in body shape between Metriaclima and Aulonocara. Each linkage group (LG, i.e.

820 chromosome) is indicated with genetic marks noted by hash marks. The phenotype related to each

821 QTL region is indicated by color. Solid bars are significant at the 5\% genome-wide level, while

822 those with white diagonals are suggestive, meeting the $10 \%$ genome-wide level. Bar widths 
823 indicate $95 \%$ confidence interval for the QTL, as calculated by Bayes analysis. Illustrations of

824 phenotypes are in Figure 2, Figure S1, and Figure S3-4. QTL scans at the genome and linkage

825 group level are in Figures S1 and S4. Details of the QTL scan including markers and physical

826 locations defining each region are in Table S1.

827

828

829 


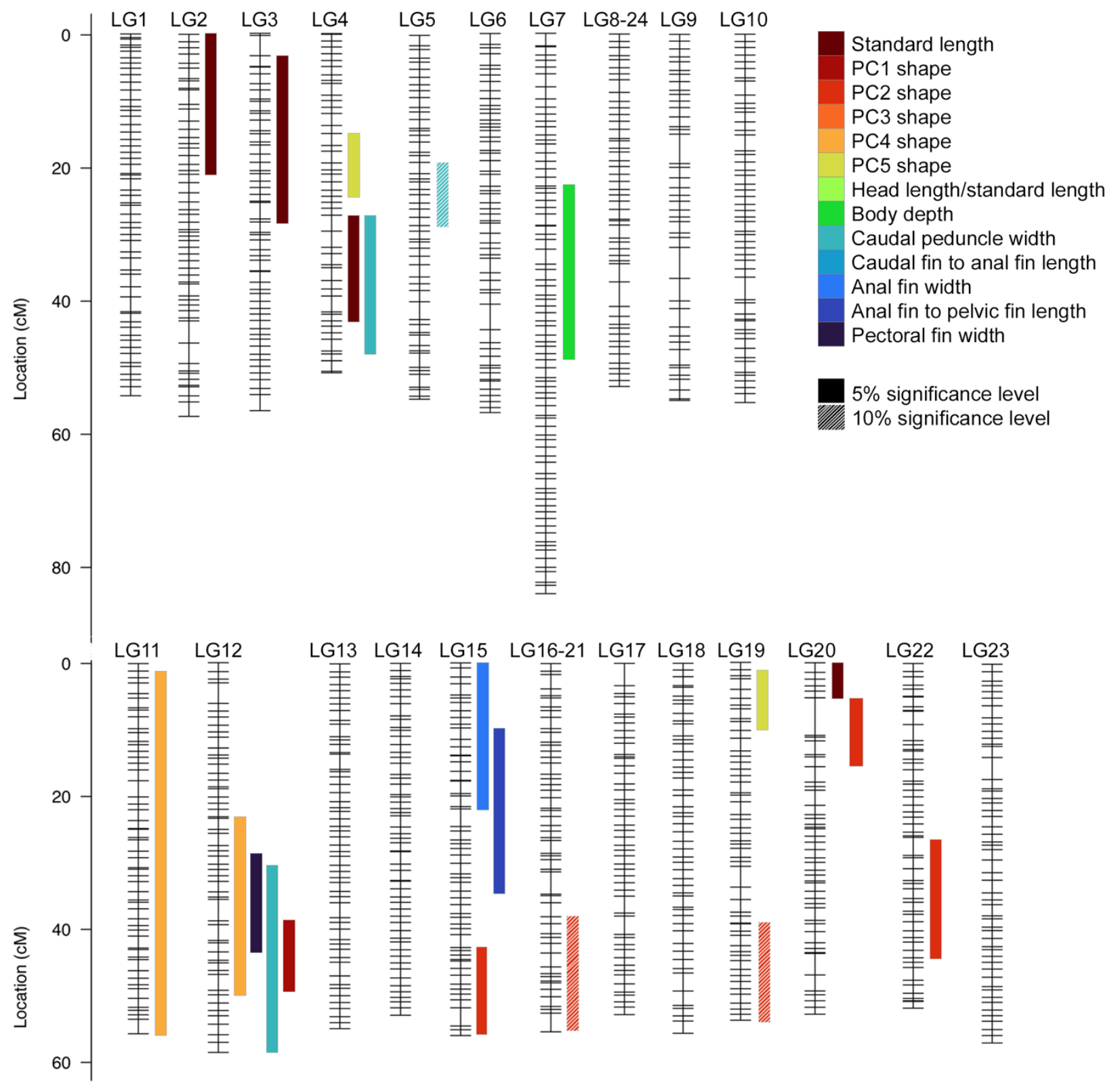

831 Figure 4. Quantitative trait loci (QTL) mapping identifies 21 intervals associated with

833 Figure 3. 

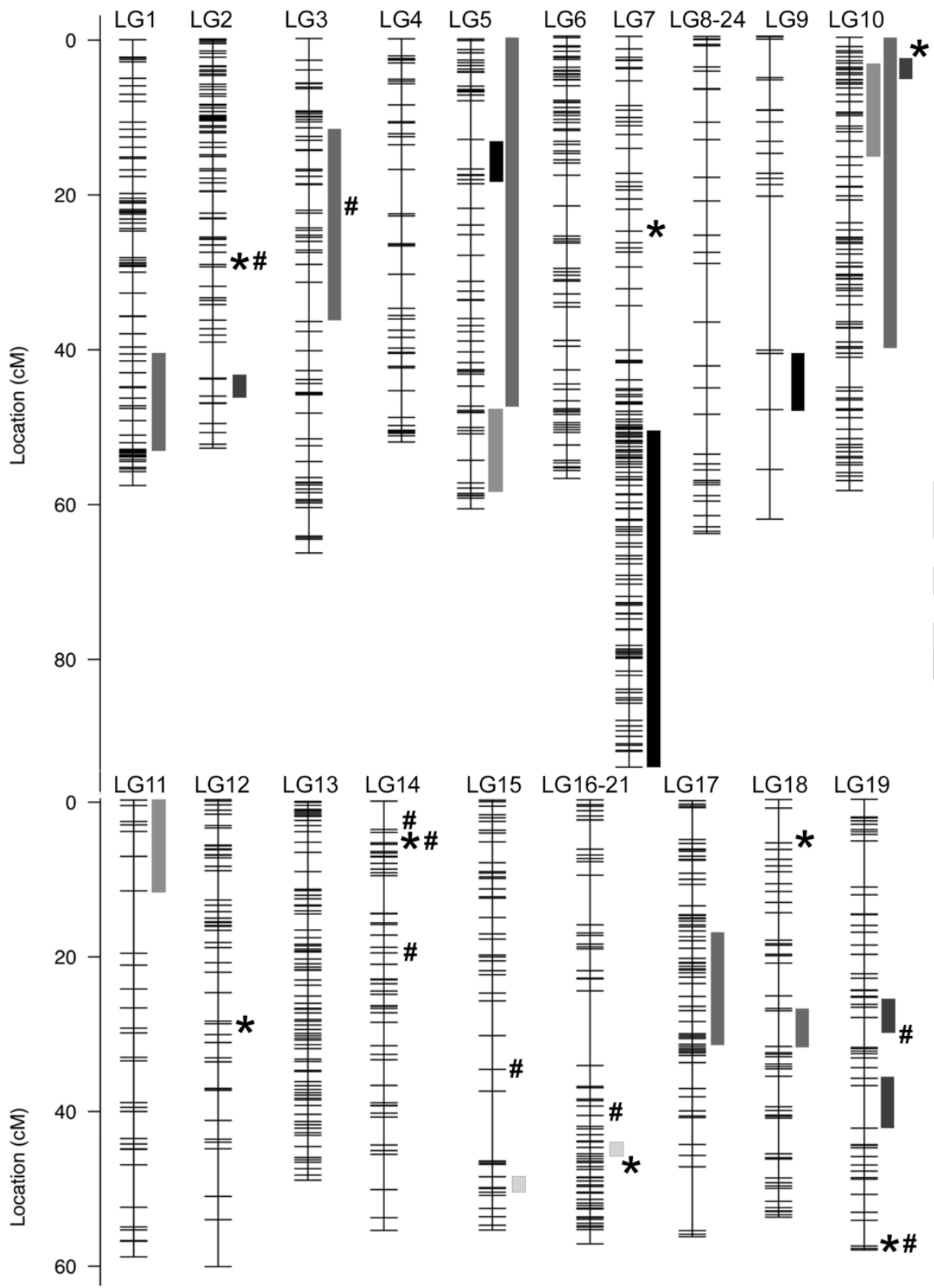

cichlids: Franchini 2014 cichlids: Navon 2017

* stickleback: Albert 2008 stickleback: Conte 2015

\# stickleback: Rogers 2012 stickleback: Yang 2016 carp: Langhari 2014
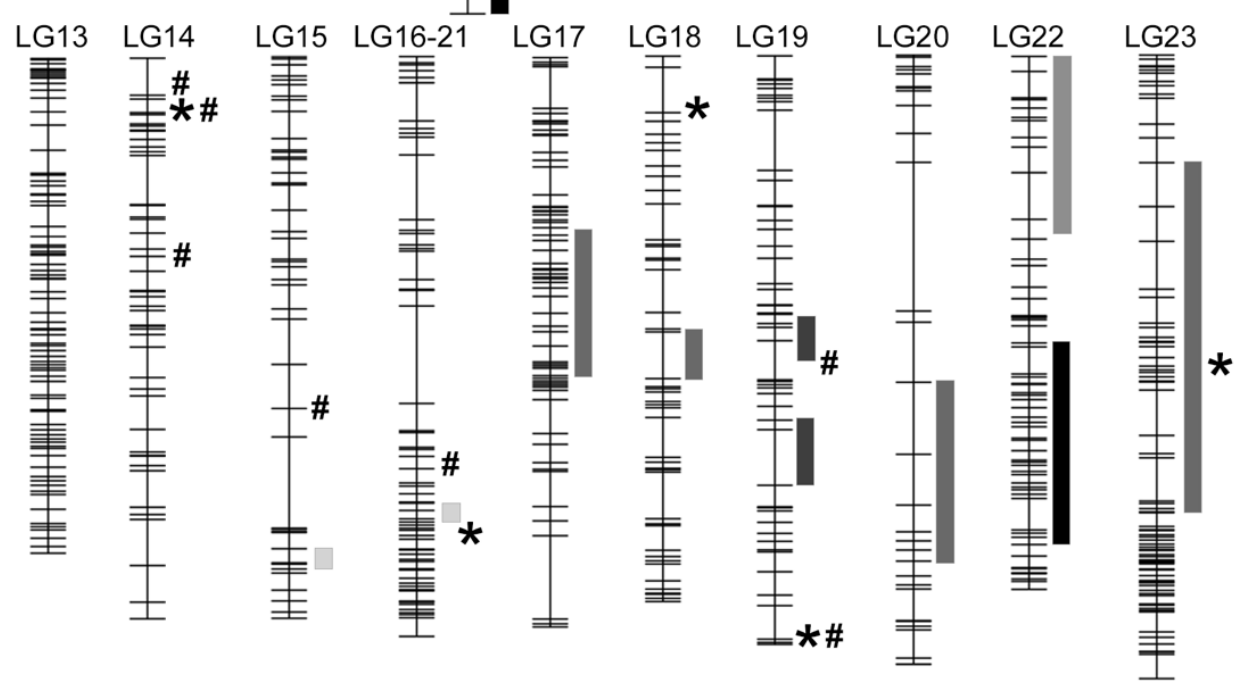

838 and carp reveals little overlap with QTL determined in this study. Reported QTL from the

839 indicated reference were converted to physical locations in the M. zebra genome UMD2a assembly

840 and mapped onto the Metriaclima x Aulonocara genetic map. Bar widths indicate the $95 \%$

841 confidence interval for the QTL as calculated by each study. Those studies that reported QTL peak 
bioRxiv preprint doi: https://doi.org/10.1101/2021.10.02.462884; this version posted October 3, 2021. The copyright holder for this preprint (which was not certified by peer review) is the author/funder. All rights reserved. No reuse allowed without permission.

842 positions, but not confidence intervals, are indicated by * and \# symbols. Details of phenotypes

843 associated with each interval, physical locations, and methods of converting to UMD2a genome

844 positions are detailed in Table S6.

845 